\title{
Modelling, Simulation and Controller Design for Hydraulically Actuated Ship Fin Stabilizer Systems
}

\author{
Alper Zihnioglu ${ }^{1}$, Melek Ertogan ${ }^{2}$, G. Tansel Tayyar ${ }^{1}$, C. Safak Karakas ${ }^{1}$, and Seniz Ertugrul ${ }^{3}$ \\ ${ }^{1}$ Naval Architecture and Marine Engineering, Istanbul Technical University, Turkey \\ ${ }^{2}$ Maritime Faculty-Marine Engineering, Istanbul Technical University, Turkey \\ ${ }^{3}$ Mechanical Engineering, Istanbul Technical University, Turkey
}

\begin{abstract}
In general, hydraulic systems that are used for ship fin stabilizers and rudders, are modelled as first or second order of linear equations to obtain only system's delay and overshoot for controlling purposes. This approach assumes the hydraulic system is well designed and contains no faults. It's an easy and quick way to focus on control subject. However, limits and capacities of hydraulic components cannot be examined carefully with this approach. Due to this deficiency, expensive over-engineered or inadequate hydraulic systems can be designed. For this reason, an interdisciplinary study was done in Istanbul Technical University. The purpose of the study is to parametrically model hydraulic system of a ship motion reduction active fin stabilizer system with fins, ship roll dynamics and controllers in detail, so every property of the system can be observed in a simulation environment via non-linear equations. With the help of parametric modelling, every component can be changed and resized easily, including the ship, fins, hydraulic components and controllers. Results obtained from simulation are verified with full scale sea trials using a ship named Volcano71.
\end{abstract}

\section{Introduction}

A ship's active fin stabilizers is an important piece of ship equipment that reduces roll motion, thus allowing better cruising experience for the people onboard and extending the service life of installed components on the ship. This equipment is hydraulically powered, because hydraulic units can have high power in small volumes making them indispensable for onboard usage. Hydraulic systems for stabilizers are generally proportional valve controlled types with constant displacement or variable displacement pumps equipped with constant speed electric motors. In recent years, there have been great interest in variable speed pump control systems [1], which offer high energy efficiency. These systems do not provide precision position control of a fin stabilizer system. Therefore, valve controlled systems are still a good choice for this kind of application. Energy efficiency can still be obtained by pressure compensated variable displacement pumps.

Rudder and fin stabilizer systems were represented by two saturating blocks and first order time delay for controlling purposes in [2]. Saturating blocks are used for limiting the desired angle and the angle rate. Delay is used to match main servo, since it is responsible for most of the delay between desired and actual rudder or fin angles. Although this is an approximately accurate assumption, there is no information about dynamic behavior of hydraulic system. To overcome this, nonlinear modelling approach was adopted for a real hydraulic fin system, installed on a motor yacht named Volcano71.

Environmental loads such as winds, waves and currents, cause a vessel to move in six degrees of freedom. These axes were standardized by SNAME (The Society of Naval Architects and Marine Engineers) in 1950. To model ship motions in detail; one needs hydrodynamic calculations of a ship that is to be modelled. These hydrodynamic calculations are made by using special naval architecture software to obtain mass, added mass, coriolis, damping and RAO (Response Amplitude Operator) matrices. This kind of work incorporates different disciplines and therefore takes too much time. Instead of this, a one degree of freedom model was used by calibrating unknown equation coefficients with known values of displacement, GM and natural roll period. Then this equation was used to obtain environmental moments as a result of experimental roll values. Fin moments were calculated in the same way by combining both theoretical and experimental results.

In the simulation, different types of controllers for hydraulic and roll motion reduction control systems were studied. The results of the simulation were verified by the real-time data from the full-scale experiments. The hydraulic controller on real system was a PID type, on the other hand roll motion reduction controller was $\mathrm{PDD}^{2}$ type. This paper, therefore, describes detailed modelling, 
simulations and controller design of hydraulically actuated fin stabilizer systems.

\section{MODELLING OF HYDRAULIC SYSTEM AND SHIP ROLL MOTION}

\subsection{Non-linear hydraulic modelling}

The hydraulic system of the ship's roll motion reduction active fin stabilizer was modelled as non-linear system. Its cost effectiveness during the initial design process became the main motivation for this approach. The hydraulic system was made of 2 asymmetric cylinders; 2 four way three position critically centered proportional valves, 2 driver cards for proportional valves, 2 potentiometers, 1 pressure compensated variable displacement pump, 1 pressure relief valve, filters, a tank and power supply. The hydraulic system scheme is given in Figure 1.

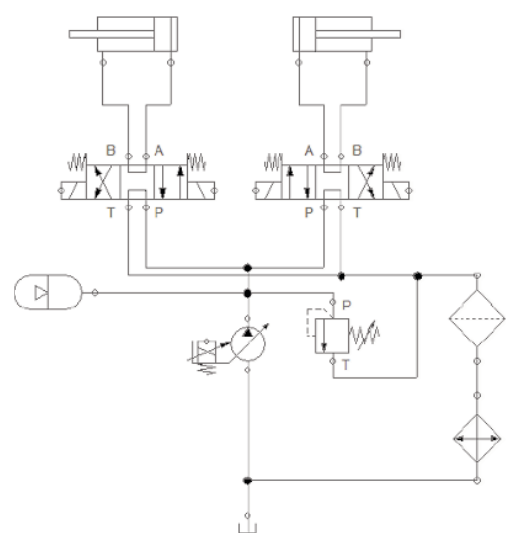

Figure 1. Hydraulic system scheme

While constructing the non-linear model, assumptions were made as follows: oil temperature is constant, pump speed is constant, hydraulic components are rigid, pump pressure-flow curve is ideal.

Pump, accumulator, pressure relief valve, proportional valve, asymmetric cylinder, cylinder friction, and dynamical change of oil bulk modulus are explained in the following sections.

\subsubsection{Pump model}

The pressure compensated pump was modelled using catalogue data and the equation given (1).

$$
\dot{P}_{S}=\frac{\beta_{e}}{V_{0}}\left[Q_{P}-Q_{R}-Q_{A}-Q_{V}\right]
$$

Since the pump was the pressure compensated type, the pressure-flow curve of the pump was rearranged for the setting of the pump's pressure. This curve for a certain pump speed is given Figure 2. In this figure $\mathrm{P}_{\max }$ refers to the setting pressure of the pump.

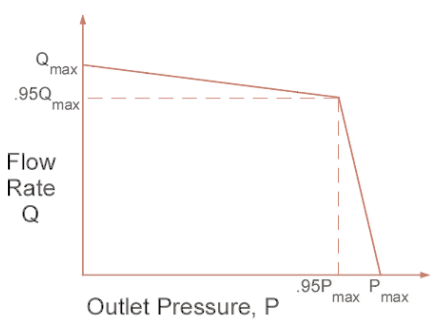

Figure 2. This curve for a certain pump speed

\subsubsection{Accumulator model}

In hydraulic systems, accumulators are used for both preventing pressure surge and providing easy pressure build up owing to the oil reserve inside. Equations given (2) - (4) were used for the modelling. An isentropic polytrophic process is assumed in these equations.

$$
\begin{gathered}
\dot{P}_{N} V_{N}^{k}+k P_{N} \dot{V}_{N} V_{N}^{k-1}=0 \\
P_{N} V_{N}^{k}=\text { Constant } \\
\dot{P}_{\text {liq }}=\frac{\beta}{V_{\text {liq }}} \frac{Q_{A}}{\left[1+\frac{\beta}{k P_{N}}\left(\frac{V_{N}}{V_{\text {liq }}}\right)\right]}
\end{gathered}
$$

\subsubsection{Pressure Relief Valve}

To mathematically model the pressure relief valve, the mass of spool, the friction coefficient between spool and the valve body, the valve spring coefficient need to be known. This kind of detailed information is not given in manufacturer's catalogue. Instead, pressure flow curves are given. By using these curves with tables and adding a bias block in a simulation, desired set pressure and flow rates were obtained. In Figure 3, an example pressureflow curve for a pressure relief valve is shown.

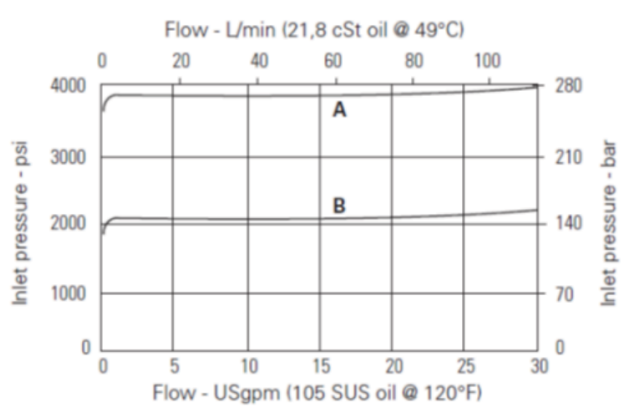

Figure 3. Pressure-flow curve for a pressure relief valve

\subsubsection{Proportional valve}

Three types of proportional valves according to spoolvalve body interaction are available on the market. These are overlapped, underlapped and critically centered. Orifice geometry also differs from valve to valve, affecting flow rate-command signal curves. It is recommended to use critically centered valves with rectangle spool geometry for better performance. Flow equations, which were used for modelling, are given (5), and (6). 


$$
\begin{aligned}
& Q_{A}=C_{d} w_{v} \sqrt{\frac{2}{\rho}} \frac{x_{\max }}{u_{\max }} u_{v}\left[\operatorname { s g } ( \operatorname { L a p } + u _ { v } ) * \operatorname { s i g n } \left(P_{s}-\right.\right. \\
& \left.P_{A}\right) \sqrt{\left|P_{S}-P_{A}\right|}-\operatorname{sg}\left(\operatorname{Lap}-u_{v}\right) * \\
& \left.\operatorname{sign}\left(P_{A}-P_{t}\right) \sqrt{\left|P_{A}-P_{t}\right|}\right]
\end{aligned}
$$

$$
\begin{aligned}
& Q_{B}=C_{d} w_{v} \sqrt{\frac{2}{\rho}} \frac{x_{s, \max }}{u_{\max }} u_{v}\left[\operatorname { s g } ( \operatorname { L a p } - u _ { v } ) * \operatorname { s i g n } \left(P_{s}-\right.\right. \\
& \left.P_{B}\right) \sqrt{\left|P_{s}-P_{B}\right|}-\operatorname{sg}\left(\operatorname{Lap}+u_{v}\right) * \\
& \operatorname{sign}\left(P_{B}-P_{t}\right) \sqrt{\left.\left|P_{B}-P_{t}\right|\right]}
\end{aligned}
$$

Another important part of the modelling is that the proportional valve and its response to a controller input. For this purpose, manufacturer's catalogues are given with bode diagrams of valve spool dynamics. In this work, given bode diagrams in the catalogue were used to construct frequency response function of spool dynamics. By using this frequency response function, transfer functions of spool dynamics were obtained utilizing system identification methods. Three identification results of signals with $5 \%, 10 \%$ and $25 \%$ amplitudes are given in Figure 4.

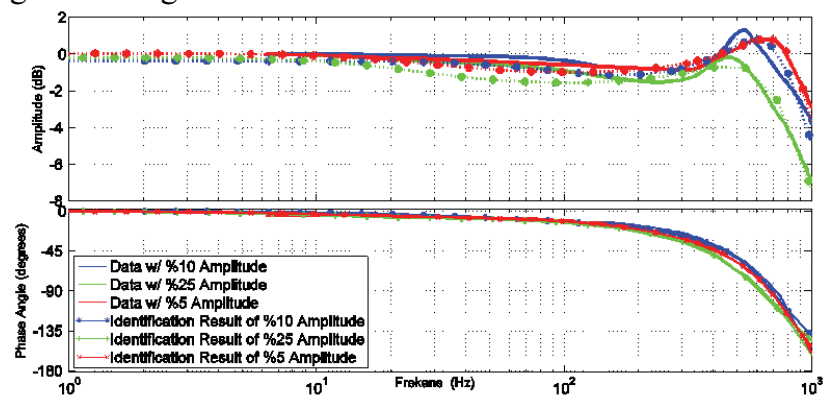

Figure 4. Pressure-flow curve for a pressure relief valve

\subsubsection{Asymmetric cylinder}

Asymmetric cylinders have non-linear nature, because of its different cap and rod side areas. A bigger cap side area creates a greater force with a slower response. On the other hand, a smaller rod side creates a smaller force with a faster response. Governing equations for asymmetric cylinder are given by (7) - (12).

$$
\begin{array}{r}
m_{t} * \ddot{x}_{y}+F_{S}\left(\dot{x}_{y}\right)+\gamma * P_{B} * A_{A}+F_{H}=P_{A} * A_{A} \\
m_{t}=m_{p}+m_{A}+m_{B} \\
m_{A}=\rho *\left[V_{A}+x_{y} * A_{A}\right] \\
m_{B}=\rho *\left[V_{B}+\alpha *\left(S-x_{y}\right) * A_{A}\right] \\
\dot{P}_{A}=\frac{\beta_{e}}{V_{A}+x_{y} * A_{A}} *\left[Q_{A}-\dot{x}_{p} * A_{A}\right] \\
\dot{P}_{B}=\frac{\beta_{e}}{V_{B}+\left(S-x_{y}\right) * A_{B}} *\left[Q_{B}+\alpha * \dot{x}_{y} * A_{A}\right]
\end{array}
$$

\subsubsection{Cylinder friction}

The non-linear friction equation is given in (13) [3]. Coefficients of this equation differ from cylinder to cylinder as a result of different diameter sizes and different chemical ingredients of seals. Exact values are obtained with tests. For this reason, the same coefficients for a similar cylinder in reference [3] were used in modelling equations.

$$
\begin{aligned}
& F_{S}=\sigma * \dot{x}_{y}+\operatorname{sign}\left(x_{y}\right) \\
& *\left[F_{\text {coulomb }}+F_{\text {static }} * e^{\left(-\frac{\left|\dot{x}_{y}\right|}{c_{s}}\right)}\right]
\end{aligned}
$$

\subsubsection{Oil bulk modulus}

Bulk modulus of oil is changed with air volume, trapped inside a hydraulic system. By increasing system pressure, the volume of air decreases and equivalent bulk modulus value converges to oil bulk modulus. With relatively small system pressures, equivalent bulk modulus makes the system harder to control by introducing a spring effect because of trapped air. Equivalent bulk modulus is calculated by using (14).

$$
\frac{1}{\beta_{e}}=\frac{1}{\beta}+\frac{V_{\text {air }}}{V_{T}} \frac{1}{\beta_{\text {air }}}
$$

\subsection{Modelling of Ship Roll Motions}

One degree of ship roll motion equation with the roll motion damping moment, generated by an active fin system, is given as (15).

$$
J \ddot{\emptyset}+\mathrm{B} \dot{\varnothing}+\Delta \overline{G M}_{T} \emptyset=\mathrm{M}_{W}+\mathrm{M}_{F}
$$

The roll motion damping moment generated by an active fin system is given in (16) [4].

$$
M_{F}=\frac{1}{2} \rho r_{c} V_{c l}^{2} A_{c} C_{L}\left(\alpha_{e}\right)
$$

$\mathrm{C}_{\mathrm{L}}$ coefficient has a non-linear condition. To take this into account, a look-up table was used in the simulation. Experimental values of $C_{L}$ coefficient for a given fin profile were used in the modelling [4].

\section{Controller Design}

Two different controllers are required to run a complete fin stabilizer system. These are hydraulic systems and roll motion reduction controllers. The hydraulic system controller in the studied project took feedback from the spool and cylinder positions, and calculated valve reference voltage between $\pm 10 \mathrm{~V}$ with PID structure. The same PID structure was constructed in the simulation and very similar results were obtained for different references. A graph including experiment and simulation results for a 5-95 mm sinusoidal reference is shown in Figure 5. The error between signals is below $1 \%$. 


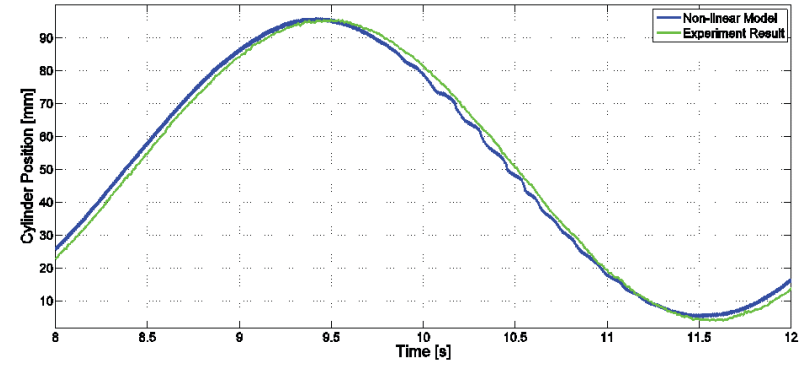

Figure 5. Comparison between the non-linear model and the experiment result for cylinder position.

A high rate of reduction in the roll motion of the ship is expected from a ship's active stabilizer fin controller. To achieve this, the roll controller must be capable of calculating suitable values of fin angle reference. There are different kinds of controller types in literature, but a conventional $\mathrm{PDD}^{2}$ structure was chosen among them to easily adopt to controller structure mentioned in [5]. General block diagram of a fin stabilizer system including ship and environmental dynamics is given in Figure 6. $\mathrm{PDD}^{2}$ controller transfer function for roll, roll rate, and roll acceleration is given by (17).

$$
Y(s)=-\left(K_{P}+s K_{D}+s^{2} K_{D 2}\right)
$$

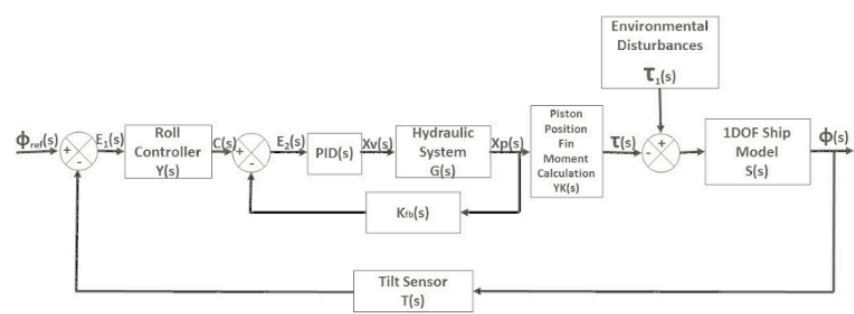

Figure 6. Block diagram of a fin stabilizer system.

\section{Experiment Results}

A hydraulic system was assembled with a pair of asymmetric cylinders having a $20 \mathrm{~cm}^{2}$ cap and $15 \mathrm{~cm}^{2}$ rod side areas with $10 \mathrm{~cm}$ stroke, a pair of four-way, three-position critically centered proportional valves, a pair of driver cards for servo controlled valves, a pair of five $\mathrm{k} \Omega$ potentiometers for measuring the angular position of the fins, 1 pressure compensated the variable displacement pump, 1 pressure relief valve, filters, and a tank. The particulars of the variable displacement pump have a maximum pressure of $\mathrm{P}_{\max }=90$ bar, and a flow speed $Q=10 \mathrm{lt} / \mathrm{min}$. The fin surface area of the stabilizer fin system is $A_{f}=0.18 \mathrm{~m}^{2}$. The electric motor power of the hydraulic system is $1.5 \mathrm{~kW}$. The assembly of the hydraulically actuated stabilizer active fin system installed on Volcano71 in length overall $\mathrm{L}_{\mathrm{OA}}=10.86 \mathrm{~m}$ is shown in Fig. 7. Full scale, sea trials were made by Volcano71. A PLC with a user interface panel was used for programming the controller in this project. It has 1 MB user memory, 1 GB secure digital memory card. Its cycle time is $1 \mathrm{~ms}$. The ship's roll and pitch angles at maximum $\pm 75^{\circ}$ were measured using a dual-axis analog tilt sensor with a resolution of $0.05^{\circ}$.

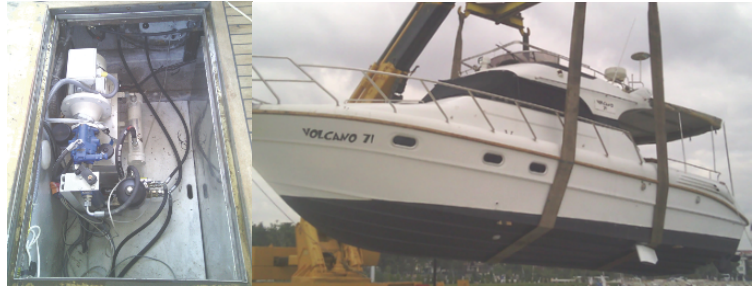

Figure 7. The fin stabilizer system's prototype was assembled on Volcano71.

The roll motion reduction controller performance was calculated via the reduction of statistics of roll (RSR), with given in (18) - (19) [6].

$$
\begin{array}{r}
R S R=100\left(1-\frac{S_{S}}{S_{U}}\right) \\
S=\operatorname{var}[\phi]
\end{array}
$$

For adjusted $\mathrm{PDD}^{2}$ coefficients, the real controller reduced the roll motion by $\% 76$. This calculation was made with consecutive time frames, representing running and idle controllers. In simulation, roll data that was collected in the idle time frame, converted to environmental moments by using (15). Then, these moment values fed back to full model, which had the same $\mathrm{PDD}^{2}$ coefficients as the real controller. A RSR value of $\% 84$ was calculated. Unstabilized roll motion values of experiment and stabilized roll motion values of simulation are given Figure 8.

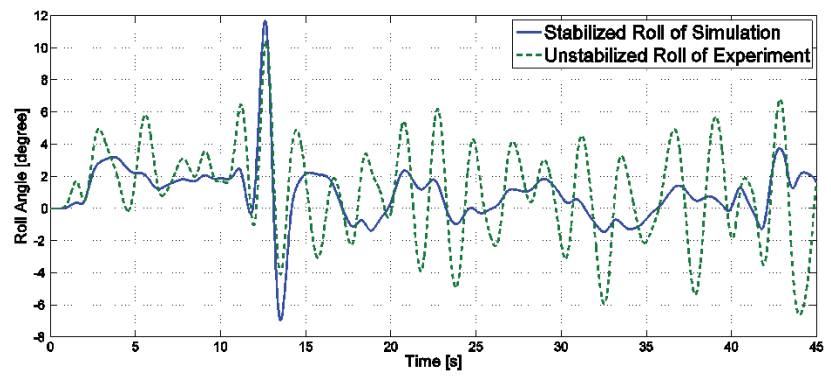

Figure 8. Roll motion reduction performance.

In addition to that, the simulation was used to solve some problems with the roll motion reduction controller. The first was the noisy feedback signal. The noise in roll feedback signal contributed wrong calculation of speed and acceleration in discrete time. The noisy roll angle signal was filtered, and a derivative calculation method was determined. These filtering, and derivative calculation methods were employed in the real system to overcome the noise problem, and satisfactory results were achieved. In the simulation, the same reference was fed through the hydraulic controller, and simulation result was compared with experimental data as shown in Figure 9 . 


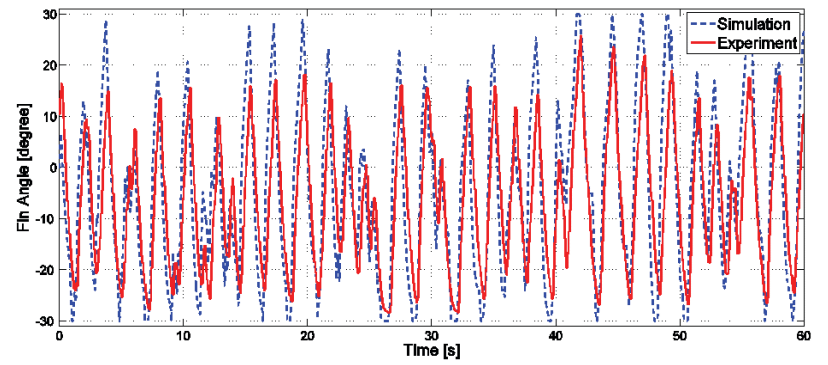

Figure 9. The comparison between simulation and experiment results of hydraulically actuated fin stabilizer position.

In the simulation, the pump pressure and the maximum flow were adjusted to find the reason for inadequate roll reduction. The possibility of inadequate pressure according to vessel speed was eliminated. The problem was identified as fast reference alteration of the roll controller caused by large roll angles according to high wave amplitudes. To solve this, the pump displacement was increased in the simulation. The hydraulic system's response was accelerated and error was reduced with higher flow rates. These results is given Figure 10.

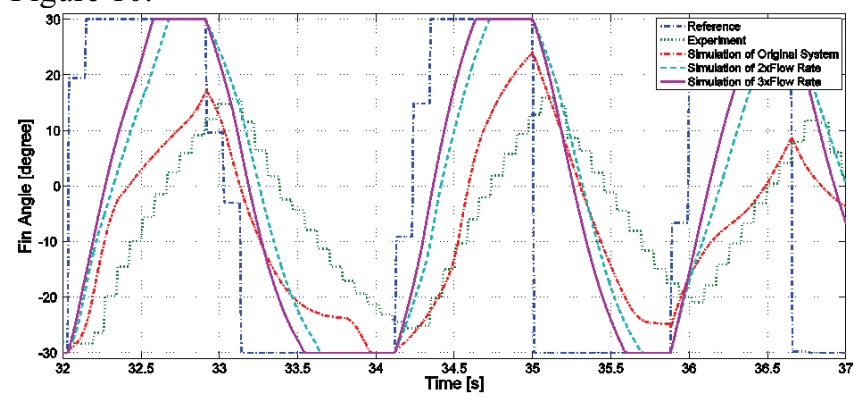

Figure 10. Results in tracking reference position of a stabilizer fin system for simulation and experimental studies.

\section{Conclusion}

In this study, a hydraulic underway fin stabilizer system including hydraulic system, ship roll dynamics, a pair of underway fins' hydrodynamic forces, and sea wave forces were modelled, so every property of the system is observed in simulation environment via non-linear modelling. With the help of parametric modeling, hydraulic components can be changed and resized easily. Ship type and properties can also be changed.

After the modelling was completed, conventional controllers of both hydraulic and roll motion reduction were designed via simulations. Controller performance can also be observed easily in the simulation.

After the installation of hydraulic components on the ship named Volcano71, full scale sea trials were done. Then, collected experiment data was compared to simulation results. Calculated reduction of statistics of roll motions was close according to the results of the model and the sea trials.

In addition to these, the simulation was used to diagnose some problems. The first of them was noisy feedback signal. The noisy roll angle feedback signal was filtered and a derivative calculation method was determined. These filtering, and derivative calculation methods were used in the real system to overcome the noise problem and satisfactory results were achieved. This model was also used to solve a problem of a stabilizer fin system's faulty tracking on a reference signal encountered in experiments.

\section{Nomenclature}

$\boldsymbol{A}_{\boldsymbol{A}}$ : Cap side area [m2]

$\boldsymbol{A}_{\boldsymbol{B}} \quad$ : Rod side area[m2]

$\boldsymbol{A}_{\boldsymbol{c}}$ : Surface area of fin

B : Roll damping moment proportional to a ship roll velocity

$\boldsymbol{\alpha} \quad$ : Cylinder volume ratio

$\boldsymbol{\alpha}_{\boldsymbol{e}}$ : Effective fin angle between flow direction and fin.

$\boldsymbol{\beta} \quad$ : Hydraulic oil bulk modulus [Pa]

$\boldsymbol{\beta}_{\boldsymbol{e}}$ : Equivalent bulk modulus [Pa]

$\boldsymbol{\beta}_{\text {air }}$ : Air bulk modulus [Pa]

$\boldsymbol{C}_{\boldsymbol{d}}$ : Discharge coefficient

$C_{\boldsymbol{L}} \quad$ : Lift force coefficient of a fin, $C_{L}\left(\alpha_{e}\right) \approx \overline{C_{L}} \alpha_{e}$ is calculated as $\left.\overline{C_{L}} \cong \frac{\delta C_{L}}{\delta \alpha_{e}}\right|_{\alpha_{e=0}}$ in general.

$\Delta \quad$ :Displacement of a ship

$\emptyset \quad$ :The ship's roll angle around the longitudinal axis

$\boldsymbol{F}_{\boldsymbol{H}}$ : External force [N]

$\boldsymbol{F}_{\boldsymbol{S}}:$ Friction force $[\mathrm{N}]$

$\boldsymbol{F}_{\boldsymbol{S}}(\dot{\boldsymbol{x}})$ : Nonlinear friction force [N]

$\overline{\boldsymbol{G M}}_{\boldsymbol{T}}$ :Transversal righting moment arm

$\gamma$ : Cylinder area ratio

$\mathbf{J} \quad$ : Mass and added mass moment of inertia

$\boldsymbol{k} \quad$ : Ideal gas constant $(\mathrm{N}: 1,4)$

Lap : Proportional valve spool lap [m]

$\mathbf{M}_{\mathbf{W}}$ : Moment of sea wave forces.

$\mathbf{M}_{\mathbf{F}}$ : Moment of an active fin system.

$\boldsymbol{Q}_{\boldsymbol{A}} \quad$ : Accumulator flow $[\mathrm{m} 3 / \mathrm{s}]$

$\boldsymbol{Q}_{\boldsymbol{P}} \quad$ : Pump flow $[\mathrm{m} 3 / \mathrm{s}]$

$\boldsymbol{Q}_{\boldsymbol{R}} \quad$ : Relief valve flow [m $\left.3 / \mathrm{s}\right]$

$\boldsymbol{Q}_{\boldsymbol{V}}$ : Proportional valve flow [m $\left.3 / \mathrm{s}\right]$

$\boldsymbol{m}_{\boldsymbol{A}}$ : Oil mass at the cap side [kg]

$\boldsymbol{m}_{\boldsymbol{B}}$ : Oil mass at the rod side [kg]

$\boldsymbol{m}_{\boldsymbol{p}}$ : Piston mass of the cylinder $[\mathrm{kg}]$

$\boldsymbol{m}_{\boldsymbol{t}}$ : Total mass [kg]

$\boldsymbol{P}_{\boldsymbol{A}}$ : Cap side pressure $[\mathrm{Pa}]$

$\boldsymbol{P}_{\boldsymbol{B}}$ : Rod side pressure $[\mathrm{Pa}]$

$\boldsymbol{P}_{\text {liq }}$ : Oil pressure in accumulator [Pa]

$\boldsymbol{P}_{\boldsymbol{N}}$ : Ideal gas pressure $[\mathrm{Pa}]$

$\boldsymbol{P}_{\boldsymbol{S}}$ : Pump supply pressure $[\mathrm{Pa}]$

$\boldsymbol{P}_{\boldsymbol{T}}$ : Tank pressure $\approx 1$ Atm. [Pa]

$\boldsymbol{r}_{\boldsymbol{c}}$ : Moment arm of an active fin system

$\boldsymbol{\rho}$ : Fluid density

$$
\boldsymbol{s g}(\boldsymbol{x}) \quad:\left\{\begin{array}{l}
x, x>0 \\
0, x<0
\end{array}\right.
$$

$\boldsymbol{S} \quad$ : Cylinder stroke length [m]

$\boldsymbol{\sigma}:$ Viscous friction term $[\mathrm{kg} / \mathrm{s}]$

$\boldsymbol{u}_{\text {max }}$ : Maximum value of the valve control signal [V]

$\boldsymbol{u}_{\boldsymbol{v}}$ : Valve control signal [V]

$\boldsymbol{x}_{\text {max }}$ : Maximum spool movement [m]

$x_{\boldsymbol{s}}:$ Spool position $[\mathrm{m}]$ 
$\boldsymbol{x}_{\boldsymbol{y}}$ : Piston position [m]

$\boldsymbol{V}_{\boldsymbol{A}}$ : Cap side volume [m3]

$\boldsymbol{V}_{\boldsymbol{B}}$ : Rod side volume [m3]

$\boldsymbol{V}_{\text {air }}:$ Air volume [m3]

$\boldsymbol{V}_{c l}$ : Relative speed with respect to flow velocity

(generally, for controller design, $V_{c l} \approx U$ is taken as the

ship speed.)

$\boldsymbol{V}_{\text {liq }}$ : Oil volume in accumulator [m3]

$\boldsymbol{V}_{\boldsymbol{N}}$ : Accumulator gas volume [m3]

$\boldsymbol{V}_{\boldsymbol{T}}$ : Total volume [m3]

$\boldsymbol{V}_{\mathbf{0}}$ : Dead volume at the pump outlet [m3]

$\boldsymbol{w}_{\boldsymbol{v}}$ : Proportional valve area gradient [m]

\section{References}

1. H. Çalıskan, T. Balkan, B.E. Platin, Hydraulic position control system with variable speed pump. In ASME, Dynamic Systems and Control Conference, pp. 275-282, (2009, January)

2. T. Perez, "Control Surfaces" in Ship motion control: course keeping and roll stabilization using rudder and fins, Springer, pp. 93-109, (2006)

3. M. Jelali, A. Kroll, Hydraulic servo-systems: modelling, identification and control, Springer, (2003)

4. T. Perez, G. C. Goodwin, Constrained predictive control of ship fin stabilizers to prevent dynamic stall. Control Engineering Practice, 16(4), pp. 482-494, (2008)

5. M. Ertogan, Roll Motion Control of Ships by Particle Swarm Optimized Adaptive PDD ${ }^{2}$ Active Fins, Ph.D. dissertation, Mechanical Eng. Dept., İstanbul Technical University, İstanbul, Turkey, (2012)

6. T. Perez, Ship Motion Performance in Ship motion control: course keeping and roll stabilization using rudder and fins, Springer, pp. 127-142, (2006) 